\title{
La crise sardinière de 1902-1913 au cœur des affrontements religieux en Bretagne
}

Jean-Christophe Fichou

\section{(C) OpenEdition}

Édition électronique

URL : http://journals.openedition.org/abpo/639

DOI : $10.4000 / a b p o .639$

ISBN : 978-2-7535-1516-1

ISSN : 2108-6443

Éditeur

Presses universitaires de Rennes

Édition imprimée

Date de publication : 30 décembre 2009

Pagination : 149-170

ISBN : 978-2-7535-1064-7

ISSN : 0399-0826

\section{Référence électronique}

Jean-Christophe Fichou, «La crise sardinière de 1902-1913 au cœur des affrontements religieux en Bretagne », Annales de Bretagne et des Pays de l'Ouest [En ligne], 116-4 | 2009, mis en ligne le 30 décembre 2011, consulté le 01 mai 2019. URL : http://journals.openedition.org/abpo/639; DOI $10.4000 / a b p o .639$ 


\title{
La crise sardinière de 1902-1913 au cœur des affrontements religieux en Bretagne
}

\author{
Jean-Christophe FicHOU \\ Professeur de géographie au lycée de Kerichen, Brest \\ CERHIO - université de Lorient
}

Sur le littoral atlantique, le monde de la sardine est secoué par des crises cycliques depuis le début de sa pêche et, plus encore depuis la mise au point de la technique de mise en conserve de ce poisson à la fin des années 1820. Les accidents dans l'histoire de cette industrie ne sont pas nouveaux; il s'en produisit notamment au cours des années 1846, 1852, 1858 ou 1870 où la pêche fut nulle ou déficitaire; ils marquent dans l'instant la vie des conserveries et s'oublient rapidement. En revanche, le premier accident de grande ampleur, s'étalant sur plusieurs années, qui touche non seulement les pêcheurs, mais aussi les conserveurs et les ouvriers des usines, commence en 1880 et s'achève en 1886 environ. Cependant, à la fin de cette période difficile les apports sont de plus en plus importants, les affaires reprennent et l'on peut même affirmer que les conserveurs connaissent alors l'apogée de leur industrie si particulière, marqué par de nombreuses constructions d'usines neuves, l'augmentation des exportations dans le monde entier et la forte hausse des bénéfices commerciaux. Hélas, après une quinzaine d'années fastes, les difficultés reviennent, pour longtemps et surtout sous plusieurs formes. Ce n'est plus seulement le manque de sardines, compensé très souvent par le travail en grand de nouveaux poissons comme le thon ou le maquereau, qui dérange un bel ordonnancement industriel déjà si souvent troublé, c'est l'affirmation de convictions fortes qui s'opposent violemment. La grande crise née à la fin de l'été 1902 est, à l'origine la simple conséquence d'une baisse des captures, mais elle devient la traduction d'un combat de nécessités et d'avantages totalement divergents dans un monde où l'affirmation des intérêts, voire des principes, ne se cache plus et se défend bruyamment. Elle se traduit comme l'inaptitude des conserveurs à modifier un comportement atavique d'intransigeance, laquelle, confrontée à la turbulente jeunesse de la revendication ouvrière, 
est inopérante. Peu à peu, les deux clans rassemblent chacun une part de l'opinion publique, et opposent, d'une certaine manière, les conserveurs et toute la fraction conservatrice de la population, aux pêcheurs et aux ouvrières soutenus par la partie la plus progressiste. Pour brouiller un peu plus les pistes, les tentatives de règlement d'un défaut d'approvisionnement se déroulent dans un climat général délétère, marqué par une très forte reprise des empoignades à caractère religieux, liée à la remise en cause du régime concordataire.

\section{Quelle crise? On peut poser la question}

Tous les observateurs de la crise sardinière s'empressent de la présenter comme telle sans analyser sérieusement les critères objectifs d'une rupture nette, réelle ou supposée, d'un cycle économique. En fait qu'en est-il de cette crise? 31759 tonnes de sardines pêchées en 1896, 44681 tonnes l'année suivante et 50908 tonnes en 1898! Jamais l'Atlantique n'a connu d'années aussi fastes; les conserveurs sont submergés par les arrivages et ne parviennent pas à travailler tous les poissons. Les stocks s'amoncellent dans les entrepôts. Les trois années suivantes, 1899, 1900 et 1901, sont encore très bonnes, mais en 1902 on note une chute puisque les pêcheurs ne rapportent que 8341 tonnes. L'année suivante n'est guère meilleure avec 9444 tonnes mais ces deux campagnes sardinières, si elles ne sont pas abondantes, sont loin d'être catastrophiques. Alors, que s'est-il passé en 1902 et 1903 pour que ces deux années deviennent synonymes de misère noire sur les côtes bretonnes et donnent naissance à un mythe toujours vivant?

"La pêche de la sardine, la principale de notre région, a été nulle en 1902 ; le poisson a fait complètement défaut. D'autre part, le prix excessif de la rogue est venu endetter le pêcheur qui s'est trouvé dans l'impossibilité de faire face à ses obligations et de subvenir aux besoins de sa famille. Par suite de la pénurie de la sardine, les ouvriers soudeurs ont dû chômer tout l'été et tout l'hiver. Leurs familles se sont trouvées plongées dans la plus grande misère. Fort heureusement la souscription ouverte pour venir en aide aux pêcheurs bretons a dépassé toutes les espérances et a permis de soulager les principales infortunes. L'industrie sardinière traverse une période critique. Les usiniers n'ayant pu fabriquer n'en ont pas moins eu à supporter des frais généraux et des impôts élevés. Ces industriels demandent un dégrèvement qui nous paraît justifié ${ }^{1}$."

\section{De multiples aléas}

En fait, il s'agit d'une succession d'incidents de pêche et de commercialisation, dont la répétition et l'accumulation portent un préjudice grave aux pêcheurs sardiniers du Finistère et du Morbihan. Pourtant cette campagne

1. Archives de la Chambre de Commerce et d'Industrie de Quimper, rapport d'activité pour l'année 1902, séance du 30 septembre 1903. 
malheureuse de 1902 n'a rien de remarquable; l'absence des sardines n'est qu'un accident cyclique dans les vicissitudes de l'existence des marins et aucun article d'aucune sorte ne s'en émeut. De plus, la situation est très différente d'un port à l'autre et la Vendée pêche normalement en 1902. D'une manière générale, il convient d'attribuer cette crise, caractérisée par deux années pauvres en poissons de toutes sortes ${ }^{2}$, au mauvais temps, aux tempêtes d'ouest et du nord-est qui tiennent la sardine éloignée des côtes bretonnes ${ }^{3}$. Les marins eux-mêmes reconnaissent ces motifs comme parfaitement valables et demandent la seule aide de leur secrétariat d'État à la Marine marchande. Certaines localités particulièrement touchées préviennent tout de même la préfecture que le manque de pêche oblige quelques marins à mendier, comme au Guilvinec où les pêcheurs « sont pour la plupart réduits à la mendicité, n'ayant même pas le pain nécessaire pour aller en mer $^{4}$ ". C'est en fait un article de l'Illustration qui alerte l'opinion et déclenche un mouvement d'ampleur nationale, sinon internationale. La direction du journal a envoyé dans le sud du Finistère, un rédacteur chargé de constater les effets d'une saison touristique désastreuse par manque de soleil. On peut cependant avancer que sa présence n'est pas fortuite ${ }^{5}$. Il est bien là pour suivre les manifestations violentes menées pour interdire les expulsions au cours du mois d'août précédent ${ }^{6}$. Toujours est-il qu'à son retour, il déclare :

"Concarneau la jolie ville malheureuse. Ce sera cet hiver la grande misère sur la côte bretonne. La saison a été mauvaise et les touristes, les baigneurs sont venus moins nombreux que les années précédentes. La pêche a été pitoyable, la pêche à la sardine surtout. Il va falloir venir en aide à tous ces braves gens si vaillants, si durs à la peine et victimes [...] Il est déjà grande misère sur la côte bretonne ${ }^{7}$."

L'information est alors reprise par l'ensemble de la presse parisienne, sans doute en mal de sujets passionnants. Le gouvernement est déjà au

2. La pêche côtière n'est pas la seule touchée; la grande pêche connaît elle aussi de graves difficultés. En 1902, les apports de morue s'effondrent, passant de 48000 tonnes en 1901 à seulement 33000 tonnes en 1902, 30000 tonnes en 1903, 1904, 1905 et 1906, avant de repartir à la hausse.

3. Service Historique de la Marine Brest, 3 P 2-11, Quimper le 3 août 1904, l'administrateur du quartier de Quimper au préfet.

4. Conseil municipal du Guilvinec, le 7 septembre 1902. Cité dans LE Coz, La Crise sardinière au pays bigouden maritime, 1902-1908, Mémoire de Maîtrise, UBO, Brest, 1995, p. 52.

5. L'Illustration publie au cours de l'été 1902 plusieurs reportages sur les manifestations organisées par la comtesse de Mun à Paris pour interdire les expulsions des congréganistes et signale la manifestation de Quimper du 22 juillet. Les choses s'enveniment encore après l'envoi de la circulaire du 29 septembre au préfet du Finistère par le président du Conseil Émile Combes et frappant de suspension de traitement les prêtres convaincus d'usage du breton dans la prédication et le catéchisme.

6. Arch. dép. du Finistère, 1 V 1152, Quimper, le 13 août 1902 relatant les manifestations des femmes concarnoises " avec le concours actif d'ecclésiastiques [...] et sous l'excitation de femmes de l'aristocratie locale ".

7. L'Illustration, le 5 octobre 1902. 
courant des misères éprouvées par les marins pêcheurs, mais il ne réagit officiellement qu'après la publication des articles des grands journaux de la capitale. La publicité tapageuse faite autour de cette histoire oblige les ministères concernés à réagir bien que pour les observateurs avertis, rien ne laisse supposer que la situation soit particulièrement critique en cette fin d'année 1902. La pêche de la sardine a connu une fin de saison précoce, c'est tout. Cependant, dès janvier 1903, le chef des services de l'Inscription maritime pour la Manche et l'Atlantique demande qu'on lui fasse connaître aussi précisément que possible la situation de tous les pêcheurs sardiniers. L'attention publique se porte sur cette population et, selon les administrateurs, il est nécessaire que le ministre demande et obtienne rapidement des aides pour venir en aide "à ces malheureux ${ }^{8}$ ". Pour les socialistes, il est évident que la faute incombe totalement aux conserveurs incapables de payer correctement les pêcheurs.

"Lorsque la pêche a donné au commencement de l'été, les usiniers ont payé si mal le poisson qu'il n'y avait aucun bénéfice. Usiniers et marchands de rogue, tous se calent, ce sont tous des exploiteurs ${ }^{9}$."

Il est certain aussi que les mesures gouvernementales sont prises très tôt pour répondre à des impératifs politiques qui prennent la forme de sollicitations toujours plus nombreuses, dont notamment celles des députés du Finistère, Georges Le Bail ${ }^{10}$ et Louis Hémon ${ }^{11}$, qui emboîtent le pas aux journaux parisiens. Le ministre ne peut pas non plus ignorer les plaintes des conseils municipaux, dont celui du Guilvinec qui précise que les habitants de la commune sont " plongés dans la plus affreuse misère; il a même tout lieu de craindre que, par suite de cette situation critique, bon nombre de personnes succombent aux suites de privations ${ }^{12}$ ".

Le ministre de la Marine n'est donc pas insensible à la situation catastrophique des pêcheurs finistériens, trop bien décrite par des maires alarmistes et une presse très misérabiliste sur le sujet. Il connaît les chiffres : dans les quartiers du Finistère-Sud on dénombre 15407 pêcheurs à la sardine. Chacun d'entre eux nourrit une famille composée de quatre à cinq personnes en moyenne. Si l'on ajoute à ce total les ouvriers soudeurs et les ouvrières d'usine, les ouvriers des chantiers navals, des fabriques de filets ou de boîtes de conserve, c'est plus de 100000 personnes qui sont susceptibles d'être atteintes par la crise dans un seul département. Le ministre, Camille Pelletan, envoie son directeur de cabinet, M. Tissier, visiter les principaux ports de pêche de ce département. Il arrive à Quimper le 16 janvier 1903

8. Service Historique de la Défense, Marine, Brest, 8 P 1-26, Saint-Servan, le 15 janvier 1903.

9. La Petite République Socialiste, le 27 février 1903.

10. Georges Le Bail, élu député radical de la $2^{\mathrm{e}}$ circonscription de Quimper en avril 1902.

11. Louis Hémon est élu pour la première fois en février 1876, député de la $1^{\text {re }}$ circonscription de Quimper. Il siège avec la Gauche républicaine. Il est réélu en 1877 et 1881, de nouveau en 1889, jusqu'en 1912, date à laquelle il devient sénateur.

12. Arch. dép. du Finistère, $10 \mathrm{M} 211$, Conseil municipal du Guilvinec, séance du 8 novembre 1903 
et part pour Douarnenez, puis Audierne, Penmarc'h, Le Guilvinec. Il rentre ensuite à Paris après avoir constaté l'étendue du désastre annoncé par voix de presse et avoir distribué quelques secours en espèces chez les pêcheurs les plus malheureux, encore une fois en présence des journalistes ${ }^{13}$. Les édiles bretons, élus de Camaret au Croisic, sont reçus par le président de la République, Émile Loubet. C'est en connaissance de cause, et sans doute poussé par "l'opinion publique ", que le ministre demande immédiatement au parlement une aide d'urgence, votée le 23 janvier 1903 pour un montant de 500000 francs; en moins d'une semaine, obtenir une telle somme relève de l'exploit.

\section{Une campagne de presse orientée}

Malgré les diverses interventions du gouvernement, assez désordonnées il faut l'admettre, la campagne de presse s'étend et les articles présentés sont de plus en plus percutants, mais de moins en moins fondés; en bref, une affaire médiatique se développe :

"Pour obtenir des souscriptions, on eut recours aux peintures les plus noires de la misère bretonne, et ces exagérations furent telles que je trouvai des Bretons ironiques disant qu'ils avaient appris la famine bretonne par les journaux de Paris ${ }^{14}$."

Il est vrai que la presse parisienne « avec une générosité d'intention des plus louables, mais aussi par un besoin de sensationnalisme et de réclame qui l'est moins, s'est laissée aller à dramatiser les choses ${ }^{15}$ ". Et pourtant les rédacteurs insistent. Au Gaulois on s'étonne de la lenteur de la réaction : " [...] c'est en janvier seulement que Paris s'émeut, que la presse fait son œuvre, alors qu'il est déjà presque trop $\operatorname{tard}^{16}$. " Dès lors, les unes des journaux se font toujours plus macabres et racoleuses :

"La Famine chez les marins pêcheur ${ }^{17}$ ",

"La Misère en Bretagne ${ }^{18}$ ",

"Sans pain et sans feu ${ }^{19}$ ",

" La grande misère de Bretagne ${ }^{20}$ ",

" [...] j'ai aperçu, autour d'une table, un homme, une femme et deux enfants qui, gloutonnement dévoraient du varech, des herbes gluantes où quelques maigres coquillages s'attachaient ${ }^{21}$."

13. Le Finistère, le 17 janvier 1903.

14. Seilhac, Léon de, La Pêche de la sardine, Paris, Masson et Gauthier-Villars, sd (1903), p. 108.

15. ROPERS, Louis, La Crise sardinière, Paris, Bonvalot-Jouve, 1906, p. 76.

16. Arch. dép. d'Ille-et-Vilaine, 4 Fg 103, Le Gaulois du dimanche du 24-25 janvier 1903.

17. Le Courrier du Finistère, le 17 janvier 1903.

18. La Vie Illustrée du 23 janvier 1903.

19. Le Monde Illustré, janvier 1903.

20. Augustin de Croze, dans la Revue, année 1904.

21. Un journaliste parisien cité dans SEILHAC, Léon de, La Pêche..., op. cit., p. 109. 
Sans doute les représentants des pouvoirs publics sont-ils dupes, mais la réalité est sensiblement autre. Cette crise n'est, encore une fois, qu'un épisode cyclique supplémentaire de la pêche de la sardine et les qualificatifs larmoyants de la presse sont inappropriés. D'ailleurs, les conserveurs ne sont guère inquiets : le ministre du commerce adresse à toutes les chambres de commerce littorales une circulaire pour connaître la situation particulière des usiniers $^{22}$. Le président de la compagnie de Brest répond :

" $1^{\circ}$ - que la disparition, en 1902, des bancs de sardines des côtes de Bretagne et de Vendée est une éventualité qui se produit parfois, sans exercer d'influence sérieuse sur l'industrie sardinière, attendu que généralement les bonnes années de pêche succèdent aux mauvaises, sans qu'on puisse en expliquer les causes.

$2^{\circ}$ - que le stock de marchandises en approvisionnement dans les usines est assez important pour assurer pendant longtemps notre commerce extérieur ${ }^{23}$."

S'il est certain que la production globale de 1902 chute par rapport aux années précédentes, les prix de la sardine fraîche, de leur côté, sont en forte hausse :

\begin{tabular}{|l|c|c|c|}
\hline & tonnage (en tonnes) & valeur (en francs) & prix au kilo (franc) \\
\hline 1899 & 28480 & 8318000 & 0,29 \\
\hline 1900 & 37098 & 10571000 & 0,28 \\
\hline 1901 & 38964 & 10620000 & 0,27 \\
\hline 1902 & 8341 & 5348000 & 0,64 \\
\hline 1903 & 9444 & 6437000 & 0,68 \\
\hline 1904 & 28091 & 20701000 & 0,74 \\
\hline
\end{tabular}

On ne peut donc parler d'une crise majeure, d'un effondrement des salaires, d'une misère noire. La sardine ne disparaît pas et seuls quelques ports sont sévèrement touchés : "Il y eut moins d'argent sur la côte; mais l'argent ne diminua pas, tant s'en faut, en proportion de la raréfaction de la sardine ${ }^{24}$. "Les pêcheurs reçoivent 7,9 millions de francs en 1898, la meilleure année, pour 51000 tonnes de sardines; en 1902, ils se partagent 6,4 millions de francs pour leurs 9500 tonnes. Le nombre d'usines finistériennes affectées à la fabrication des sardines à l'huile et celui des ouvrières ne varient pas non plus. On compte 97 usines et 8657 employées pour la saison 1902; elles sont 96 et 8496 respectivement l'année suivante ${ }^{25}$. En

22. Ministère du Commerce, circulaire du 21 février 1903.

23. Archives de la Chambre de Commerce et d'Industrie de Brest, lettre du 18 avril 1903 du président Marfille au ministre du Commerce.

24. Vallaux, Camille, La Basse Bretagne. Étude de géographie humaine, Paris/Genève, Slatkine, 1980, (1906), p. 248

25. Archives de la Chambre de Commerce et d'Industrie de Quimper. Quimper, rapports d'activité, situation industrielle et commerciale 1902 et 1903. 
1904, alors que sardine revient, les chiffres, encore une fois, sont similaires, 94 et 8620 . Pour les ouvrières, en revanche, la situation est beaucoup plus difficile puisque payées au mille de poissons travaillés, elles voient leurs ressources globales décroître dangereusement. Les salaires versés en 1898 s'élevaient à 9,1 millions de francs, mais ils ne sont plus que de 1,7 million en $1903^{26}$. Pour les fabricants, par contre, rien n'a été modifié; pour eux il s'agit d'un accident conjoncturel sans gravité particulière, redouté mais attendu avec une relative quiétude dans la mesure où les stocks sont au plus haut. Pourtant, les cas de faillites, rares rappelons-le, sont amplifiés au fil des mois, dramatisés et utilisés par les fabricants pour forcer le gouvernement à les écouter et à accepter leurs solutions pour résoudre cette crise. En pays bigouden, la maison Paul de Penanros ${ }^{27}$ est en liquidation judiciaire alors que les fabricants bordelais Dandicolle et Gaudin sont obligés de vendre leurs usines à un groupe anglais ; immédiatement la chambre de commerce de Quimper s'inquiète de ces fermetures et réclame au gouvernement des mesures rapides, dont la liberté entière de pêche ${ }^{28}$.

De toute manière, crise ou non, il demeure une certitude : la vie des pêcheurs sardiniers est déjà difficile quand la campagne est bonne, quand ils se privent simplement du superflu, si bien que le moindre accident est vécu comme une catastrophe car elle entraîne la misère et " la privation du nécessaire ${ }^{29}$ ". La crise de 1902 n'est pas la première du genre, mais c'est la première dont « le reportage se soit emparé, la première, par conséquent, dont on ait entendu parler loin des côtes de l'Ar-Mor ${ }^{30}$ ". On recherche le cas particulier et désespéré; on répète les mêmes erreurs en les amplifiant; on dramatise la situation; on grossit des faits déformés; on invente des situations épouvantables. Les marins pêcheurs eux-mêmes se prêtent au jeu; les conserveurs reprennent les faits en les déformant, contribuant ainsi à renforcer la légende. Hubert Ouizille rappelle, en 1926, que pour l'année 1902, la fabrication française tombe " de 1000000 de caisses à 196000 et encore ce chiffre n'a été atteint que parce qu'à Belle-l̂le et Quiberon la pêche est restée pendant toute la saison régulière et fructueuse $\mathrm{e}^{31}$ ".

26. ROBERT-MULleR, Charles, Pêches et pêcheurs de la Bretagne atlantique, Paris, Masson, 1944, p. 100

27. Arch. dép. du Finistère, 65 U 14-20, Faillites, 1907.

28. Archives de la Chambre de Commerce et d'Industrie de Quimper, Quimper, séance octobre 1907.

29. BLoy, Léon, Le Sang des pauvres. Paris, Gallimard, 1948 (1909) p. 39.

30. VAllaUX, Camille, La Basse Bretagne... op. cit., p. 248.

31. OuzILLE, Hubert, Les Conditions économiques actuelles des industries sardinières française et portugaise. Thèse de doctorat, 1926, p. 14. Les chiffres avancés sont sujets à caution. D'une part, nous ne possédons aucune statistique sûre concernant la production totale nationale et, d'autre part, si l'on s'en tient aux seules exportations, nous constatons que la France envoie à l'étranger 9960 tonnes de sardines à l'huile en 1901, 9960 en 1902 et encore 6380 tonnes en 1903. Il est impensable dans ces conditions que la production totale ait diminué de $80 \%$. 


\section{Une situation maintes fois vécue mais relayée pathétiquement}

Ce que l'ensemble des Français découvre en janvier 1903 est un simple accident halieutique, accentué par un malaise beaucoup plus ancien et plus profond. Cependant, deux motifs permettent d'expliquer les exagérations relevées par Vallaux, éditoriales selon lui, mais, je rajouterai, aussi parlementaires. D'une part, certaines communes, comme Audierne, ont connu un réel drame; on y débarque à peine 13 tonnes de sardines en 1902, soit 17 fois moins que l'année précédente, production bien insuffisante pour nourrir les pêcheurs locaux qui ne pratiquent que cette activité alors que le maquereau est présent en nombre dans la baie. La presse s'empare de cet exemple particulier et tend à généraliser un problème local à l'ensemble du littoral ce qui, bien entendu, est erroné.

" N'a-t-on pas été jusqu'à affirmer au ministre que la flottille d'Audierne qui comprend plusieurs centaines de bateaux n'a pas pêché au total depuis l'ouverture de la campagne un millier de sardines. L'exagération est manifeste; elle est cependant l'indice d'une affreuse disette de poisson ou du moins, la preuve que nos marins bretons ne savent pas le capturer [...] leurs habitudes, leurs idées témoignent d'une naïveté et d'une étroitesse d'esprit incroyable ${ }^{32}$."

D’autre part, les années précédant la « crise " sont prospères, si bien que de nombreux petits paysans et ouvriers agricoles abandonnent à l'époque leur campagne pour tenter leur chance sur la mer. En 1902, on atteint le chiffre maximum d'inscrits maritimes jamais relevé en France, mais parmi eux bon nombre sont des néophytes, des déracinés issus des campagnes voisines surchargées, incapables d'envisager une autre pêche que celle de la sardine, la plus facile à réaliser. Il leur est impossible d'armer des navires plus puissants, car ils sont trop pauvres, ou de s'engager sur des bâtiments hauturiers car ils sont trop inexpérimentés. Coupés du monde rural et inadaptés au monde maritime, ils forment un groupe fragile frappé de plein fouet dès l'arrivée des premiers mauvais jours.

"En dix ans le nombre des bateaux et des pêcheurs a presque augmenté d'un tiers. Quantité de journaliers agricoles, voire de petits fermiers ont abandonné les champs pour le large. Il y a beaucoup trop de pêcheurs sardiniers en Bretagne et on commence à s'en apercevoir ${ }^{33}$. "

Enfin, les observateurs unanimes critiquent la passivité des pêcheurs. Il apparaît aux yeux de tous qu'il faut diversifier les pêches. On ne peut jouer sa saison et son existence sur une seule espèce, le pari est trop risqué, d'autant plus que la sardine est trop souvent absente au rendez-vous.

"[...] La sardine a déserté nos côtes : or c'est la seule ressource de toute la population maritime de Camaret, Douarnenez, Audierne, Penmarc'h, Guilvinec, Lesconil, Concarneau, etc. Sur le littoral du Morbihan, la misère est grande aussi mais moindre cependant car les marins de ces parages

32. Le Yacht, 1906, p. 625 .

33. LE GofFIC, Charles, dans l'Éclair du 13 octobre 1903. 
trouvent encore quelques ressources dans la pêche de produits consommés frais $^{34}$."

Et puis les ecclésiastiques locaux n'oublient pas de rappeler aux pêcheurs bretons que le « petit père Combes " n'a pu affronter impunément Dieu sans que ce dernier ne se fâche. La sardine a disparu par la volonté divine et s'est enfuie pour punir la "fille aînée de l'Église " devenue sans doute trop laïque. "Simple coïncidence, peut-être dirions-nous, mais bien faite pour intriguer et faire réfléchir ${ }^{35}$. " Pour le curé de Concarneau, la catastrophe est bien une épreuve infligée par Dieu et il décide d'organiser un pèlerinage à Sainte-Anne-de-Fouesnant ${ }^{36}$. La sardine devient clairement l'objet d'enjeux politiques; le groupe qui parviendra à sauver les marins pêcheurs obtiendra leurs voix. Les évêques interviennent directement dans le débat pour faire barrage à l'élection des candidats radicaux et de leurs alliés. "En votant pour les libéraux et les patriotes contre la francmaçonnerie, au scrutin de ballottage, les catholiques de France voteront contre l'étranger ${ }^{37}$. " L'évêque de Quimper, Monseigneur Dubillard, est très explicite puisqu'il demande aux paroissiens de " toujours choisir parmi les candidats ayant des chances de succès, le catholique qu'il jugera le plus apte par sa sagesse et sa modération à défendre efficacement les droits de la conscience et de la religion ${ }^{38}$ ". Mais rien n'y fait, et finalement les catholiques sont obligés d'accepter le nouveau ministère formé par Émile Combes, dont l'un des principaux combats est la lutte contre le cléricalisme. De leur côté, les aides apportées aux marins en difficulté par l'Église sont toujours accompagnés d'un message clair de l'évêque, relayé par les prônes des curés dans toutes les paroisses :

"Quant à vous pêcheurs de la côte, qui avez été si généreusement secourus, vous vous souviendrez de vos bienfaiteurs et vous prierez pour eux [...]. Dans les luttes présentes, que vous restiez fermes dans la foi et qu'en toutes circonstances vous soyez disposés à faire pour elle les plus grands sacrifices $[\ldots]$. Vous ne faillirez point à vos patriotiques et religieuses traditions ${ }^{39}$. "

Dans le Finistère et le Morbihan, les prêtres et leurs évêques sont soutenus dans leur combat par les conserveurs parfois très virulents et qui

34. Le Yacht, le 24 janvier 1903.

35. L'Action Libérale, cité dans Boulard, Jean-Claude, L'Épopée de la sardine, un siècle d'histoire des pêches, Rennes, Ouest-France, 1991, p. 25.

36. Gú́guen, Michel et Le MaITRE Louis-Pierre, Matelots de Concarneau, 1800-1914. Concarneau, à compte d'auteur, p. 286.

37. La Semaine Religieuse de Saint-Brieuc et de Tréguier, 2 mai 1902, p. 277, cité dans BELCEIL Dominique, "Les évêques bretons face à la loi de séparation des églises et de l'État (1902-1936) ", dans THEURET, Johan (dir.), Un Siècle de laïcité en Bretagne 1905-2005, Rennes, Éditions Apogée, 2005, p. 41.

38. Arch. dép. du Finistère, 1 V 86, Lettre pastorale de Monseigneur Dubillard, Quimper, le 23 janvier 1902. À la mort de monseigneur Valleau, évêque de Quimper, c'est le vicaire général du diocèse de Besançon, François-Virgile Dubillard qui est nommé par décret du 7 décembre 1899 pour le remplacer.

39. Arch. dép. du Finistère, 1 V 38, Lettre pastorale de Monseigneur Dubillard, Quimper, février 1903. 
ne se contentent pas de simples processions ou de création de patronages. Ainsi, après l'adoption de la loi du premier juillet 1901 sur la suppression des congrégations, ils exercent des pressions sur leurs ouvriers pour empêcher les agents du gouvernement d'intervenir dans les bâtiments religieux. Ainsi à Concarneau, Cabon, gérant d'usine, Chancerelle Alfred, Veuve Lacaze, Balestrié, Le Marié, de Penanros, Billette de Villeroche, fabricants de conserves " qui avaient pris une part active à la résistance, avaient engagé leur personnel à se porter en masse aux abords du couvent le jour où les agents du gouvernement viendraient mettre à exécution le décret concernant les congrégations religieuses enseignantes ${ }^{40}$ ". À Douarnenez, Gustave de Pennanros et Robert Chancerelle sont aussi à la tête des manifestants; le second est condamné pour " outrage envers un commissaire spécial ${ }^{41}$ ". En 1904, les résultats de la pêche sont excellents et de nombreux marins pêcheurs " dans leur reconnaissance, attribueraient ce résultat à la protection de la Vierge du Raz " inaugurée en juillet $^{42}$.

L'année 1905 est de nouveau mauvaise pour les pêcheurs sardiniers. Cependant la campagne de presse ne prend pas d'ampleur malgré quelques titres accrocheurs qui retrouvent la une des journaux à la veille du vote de la loi de séparation de l'Église et de l'État dont la discussion est prévue en décembre :

"Comment trois cent mille Français sont cette année réduits à la famine ${ }^{43}$ " "Au pays de la faim ${ }^{44}$ "

La presse religieuse régionale n'est pas en reste et si la sardine est de nouveau absente, il semble bien évident que cette disparition soit liée au projet de loi de séparation. Le vote définitif, le 9 décembre 1905, plonge les catholiques dans la consternation et confirme les craintes exprimées quant à la conscience religieuse des sardines. Conscience bien versatile puisqu'elles sont de retour en 1906, année d'élections législatives au cours de laquelle le républicain Le Bail doit affronter l'avocat Henri Beauchef de Servigny, cousin et neveu de conserveurs dont les votes lui sont acquis. Et pour remporter la victoire, le candidat conservateur peut compter sur le soutien des membres de la Ligue Patriotique des Femmes françaises qui compte un certain nombre d'épouses de marins chargées de les inciter

40. Arch. dép. du Finistère, 1 V 1164. Concarneau, le 11 octobre 1902, le commissaire au préfet.

41. Arch. dép. du Finistère, 1 V 1152, Tribunal correctionnel de Quimper, le 13 septembre 1902.

42. Arch. dép. du Finistère, 1 V 38, Lettre pastorale de Monseigneur Dubillard, Quimper, le 23 décembre 1904. La statue de la Vierge est un groupe monumental de 11 tonnes offert par le sculpteur Godebski en souvenir de son fils mort au Tonkin.

43. La une du Matin du 2 septembre 1905.

44. Le Matin du 29 novembre 1905. Ce titre sera repris à de très nombreuses occasions jusqu'en 1913. Maizeroy, René, dans les Annales Politiques et Littéraires du 9 février 1913, $\mathrm{n}^{\circ} 1546$. 
à négliger le candidat " séparatiste ${ }^{45}$ ". Il n'est plus question d'une aide charitable mais bien de pressions électorales, somme toutes banales à l'époque. Malgré les efforts du clergé, malgré les mauvaises pêches, malgré l'absence de la sardine, Le Bail retrouve sa députation. Le dépit de l'évêque est réel :

«Nous vous conjurions de réfléchir et d'agir virilement afin d'envoyer dans nos Chambres françaises des hommes capables d'enrayer le mal et sauver chez nous l'existence même de la religion et du culte catholique. Hélas! Notre voix n'a pas été entendue et Nos prières sont restées sans effets ${ }^{46}$. "

On ne peut cependant se voiler la face et la situation déjà fragile des populations littorales devient de plus en plus difficile, quel que soit leur métier qui est de toute manière lié de près ou de loin au monde de la conserverie. Les marins sardiniers de toute la Bretagne méridionale ne peuvent plus se satisfaire de gains dérisoires. Au cours de l'été 1907, entre juin et novembre, les pêcheurs concarnois ont gagné " à peine cent vingt à cent cinquante francs à l'homme ${ }^{47}$ ".

"Les bateaux sardiniers désarment tous, le mauvais temps qui dure depuis plusieurs jours les empêchant de sortir. Jamais au dire des plus vieux marins, l'on avait vu une année aussi désastreuse. Quelques milles de sardines, seulement, ont été pêchées en baie de Concarneau, d'ordinaire si fructueuse, pendant la campagne qui vient de se terminer ${ }^{48}$."

Les ouvriers boîtiers et les femmes d'usine sont encore plus éprouvés car la majorité des usines n'a pas ouvert, ou seulement quelques semaines, au cours de l'été. Les commerçants locaux ont voulu « aider leurs frères et leurs camarades de travail de toute leur volonté en leur faisant un crédit et tout est resté impayé ${ }^{49}$ "; les dossiers de faillite des boulangers finistériens ouverts durant cette période l'attestent ${ }^{50}$. Les autorités s'inquiètent et demandent de multiples enquêtes aux préfets et commissaires pour connaître l'ampleur des dégâts.

"Au cours de la tournée que j'ai effectuée dans le canton de Crozon, j'ai pu constater que la misère était extrême parmi les nombreuses familles de pêcheurs. Toutes sont chargées d'enfants, souvent en bas âge; et la pénurie complète de poissons, qui dure depuis plusieurs années, a plongé tous ces malheureux dans la plus pénible situation ${ }^{51}$."

45. Arch. dép. du Finistère, 3 M 301, Quimper, le 27 février 1906, le préfet souligne que le mouvement est "fortement implanté dans le Sud Finistère en faveur des candidats catholiques".

46. Arch. dép. du Finistère 1 V 38, lettre pastorale de Monseigneur Dubillard, Quimper, le 2 février 1907.

47. Arch. dép. du Finistère, 10 M 211, Concarneau, le 28 novembre 1907, pétition des marins adressée au conseil municipal.

48. Arch. dép. du Finistère, 18 J 24, Le Progrès du Finistère, le 25 octobre 1907.

49. Arch. dép. du Finistère, 10 M 211, Concarneau, le 28 novembre 1907.

50. Arch. dép. du Finistère, 65 U 14-15, 16, 17, 18.

51. Arch. dép. du Finistère, 10 M 21, Châteaulin, le 13 décembre 1907, le sous-préfet au préfet. 
La crise de la sardine devient une affaire nationale, une grande cause, qui intéresse et passionne même toute la population française. Elle occupe les bancs de la Chambre des députés aux cours de nombreuses séances et les élus des circonscriptions littorales et sardinières sont très présents dans l'hémicycle pour rappeler les souffrances de leurs administrés, dans de longs débats souvent sans fin. Pourtant, les gouvernements successifs ne parviennent pas à régler le problème, quelle que soit leur coloration politique et malgré toute leur bonne volonté. Les ministères successifs ${ }^{52}$ sont incapables d'apporter une réponse claire aux problèmes des pêcheurs et des conserveurs.

\section{Les moyens de résoudre la crise}

La première raison invoquée pour expliquer la disparition du trésor halieutique, et sans doute la plus facile à admettre pour l'époque, est d'ordre religieux. Les conserveurs, et une bonne partie de la population, affirment que cette désertion de la sardine est une punition divine :

" Nous devons constater que c'est depuis 1902, date à laquelle nos marins pêcheurs crurent devoir élire leur "blocard" et sectaire Le Bail, député socialiste, que le mal cruel dont ils souffrent a commencé. Simple coïncidence, nous dirons les adversaires. Simple coïncidence, peut-être dirons-nous, mais bien faite pour intriguer et faire réfléchir. "

Et le recteur de Douarnenez de renchérir : "Vous avez fui les bancs de l'Église alors les bancs de sardines ont fui la côte ${ }^{53}$. "Le maire de Concarneau, Billette de Villeroche et son adjoint Le Marié, tous deux conserveurs, se plaisent à rappeler la concordance de dates entre celle des expulsions des filles du Saint Esprit au cours du mois d'août et celle des premières pêches dérisoires ${ }^{54}$. De toute manière, pour tous, il est clair qu'il s'agit d'un problème si complexe à résoudre, dont les termes sont si contradictoires, et les intérêts en présence si opposés, " que les efforts de chacun n'ont guère abouti qu'à montrer à quel point il est puéril de méconnaître certaines lois économiques et de chercher dans leur seule violation un remède qui, s'il n'était illusoire, serait encore plus dangereux que le mal qu'on se propose de guérir ${ }^{55}$ ".

Mais tous, fabricants ou pêcheurs, ont-ils agi au mieux des intérêts communs? Ont-ils tenté de se comprendre? L'attitude des usiniers n'est guère limpide.

52. Combes, Rouvier, Sarrien, Clemenceau, Briand 2, Monis, Caillaux, Poincaré, Briand 3, Barthou, Doumergue, Ribot et Viviani.

53. L'Action libérale, du 24 mars 1906, cité dans LuCAS, Maurice, Luttes politiques à Douarnenez, 1890-1925, Morlaix, Imprimerie nouvelle, 1975, p. 92.

54. Les deux fabricants sont suspendus de leurs fonctions pour leur participation aux manifestations par les arrêtés préfectoraux des 2 et 3 septembre 1902, Le Finistère du 10 septembre 1902.

55. Rapport de l'inspecteur général des Pêches, Fabre-Domergue, Paris, le 10 mars 1907. 
"Qu'on fait les fabricants? si les pêcheurs ont une grande méfiance visà-vis des fabricants, ces derniers ont parfois vis-à-vis les uns des autres une défiance qui serait risible si elle n'avait pas d'aussi triste conséquence pour leur industrie en général. Pour justifier leur inertie et continuer à ne rien faire les uns prétendent qu'il suffit de se fier à la Providence qui, ayant fait disparaître la sardine, saura bien la faire revenir; d'autres disent que, le Gouvernement ayant promis une enquête, il n'y a qu'à en attendre les résultats et quelques-uns profitent de la pénurie de la sardine pour se livrer aux fraudes les plus variées, ce qui leur permettra, pensent-ils, d'attendre des jours meilleurs ${ }^{56}$."

Les pêcheurs, de leur côté, insistent pour obtenir des prix élevés des quantités de sardines qu'ils débarquent. Ils ne veulent pas s'outiller pour pouvoir se rattraper par la quantité sur un prix de vente relativement peu élevé.

"L'objection de la pauvreté individuelle du pêcheur l'empêchant d'acquérir des filets plus chers ou le moteur qui l'affranchira de l'emprisonnement des calmes, est depuis longtemps prévue et l'établissement du crédit maritime a pour but de résoudre cette grosse difficulté [...] Dans le domaine économique, qui ne progresse pas est destiné à périr ${ }^{57}$. "

Il est clair que pour bon nombre des observateurs le moyen essentiel pour combattre le mal lié à la pénurie de sardine réside bien dans le changement radical de la nature des pêches et donc du comportement des pêcheurs. Il faut que ceux-ci abandonnent leurs bateaux et des engins inadaptés et se « livrent à la grande pêche au large sur des navires pontés qu'ils feraient construire ${ }^{58}$ ". Certains observateurs préconisent des solutions encore plus difficiles à mettre en place mais qui répondent à une réalité tangible : " L'école et le crédit! voilà ce qu'il faut à nos marins pêcheurs et quand vous leur aurez donné cela le problème social ne se posera plus ${ }^{59}$." Et puis tous les acteurs contemporains n'ont pas cette vision catastrophiste de la crise sardinière. Le commissaire spécial de Quimper chargé d'étudier et de surveiller les événements est au cœur du problème, mais pour lui, bien des faits sont exagérés. Il note que dix ans après la chute brutale des approvisionnements, il ne compte que très peu de fermetures d'usines et encore moins de faillites. À l'inverse, il note la création de plus d'une dizaine d'usines : pour lui la situation n'a pas changé, tout au moins en ce qui concerne le nombre total des établissements ${ }^{60}$.

56. Madurec, Yves, La Crise sardinière 1902-1904. Paris, imp. Dupont, 1904, 15 p.

57. VERO, Max, « La Crise sardinière ", dans le Yacht 1913, p. 72.

58. Arch. dép. du Finistère, 10 M 21, Châteaulin, le 13 décembre 1907, le sous-préfet au préfet.

59. Le Bail, Chambre des Députés, séance du 3 décembre 1909, Discussions sur la crise sardinière.

60. Arch. dép. du Finistère, 4 S 359, Quimper, décembre 1912, le commissaire spécial. 


\section{Les aides financières}

De toute manière, pour tous les marins en difficulté il est évident que l'État protecteur va intervenir pour soulager leurs maux, comme d'habitude; n'est-il pas le " dispensateur de la manne des subventions et des secours, [...], c'est le protecteur riche et puissant qui apporte l'argent dont on a besoin ${ }^{61}$ ".En effet, au début du mois de décembre 1902, les députés du Finistère Le Bail et Hémon, et leur confrère morbihannais, Guieyesse, présentent une proposition de loi à la Chambre en faveur des marins pêcheurs "éprouvés par l'insuffisance des résultats de pêche ". Ils demandent un secours de 2 millions de francs ${ }^{62}$. Dès lors des aides de toutes parts et de tous montants parviennent en Bretagne pour tenter d'apporter un peu d'espoir et de réconfort aux marins sardiniers. Des souscriptions sont ouvertes par les principaux journaux de la région, dont la Dépêche de Brest qui annonce fièrement avoir récolté plus de 15000 francs dès la fin du mois de janvier ${ }^{63}$. Pour Le Courrier du Finistère, le crédit demandé au gouvernement " ne pouvant être accordé en temps utile - s'il l'est jamais - pour soulager la misère qui s'est abattue sur nos pêcheurs ${ }^{64}$ ", il faut lancer un vaste programme de souscription. Le mouvement de générosité se développe rapidement dans toute la France. Partout dans le pays, les bonnes volontés se mobilisent et notamment les chambres de commerce maritime. Pour le président de l'assemblée brestoise,

" En présence de la profonde misère qu'éprouvent les marins pêcheurs de notre littoral ainsi que leurs nombreuses familles, par suite du manque absolu de pêche, il est urgent aujourd'hui, de trouver des sommes assez importantes afin de soulager le plus promptement possible, ces grandes infortunes ${ }^{65}$."

Un secours de 500 francs est voté en conséquence, la même somme qu'envoyée quelques semaines plus tôt aux rescapés de Saint-Pierre à la Martinique, c'est-à-dire très peu. La chambre de Saint-Brieuc adresse en mars une somme de 100 francs en vue de venir en aide aux pêcheurs de sardines, à peine le dixième du revenu annuel d'un pêcheur. Celle de Dunkerque adresse un chèque de 500 francs " en vue de contribuer à atténuer les cruelles épreuves que subissent les marins pêcheurs bretons". La chambre de Fougères envoie un mandat poste de 50 francs " en vue de venir en aide aux malheureux pêcheurs bretons ".

À leur tour, de nombreux journaux participent à cette collecte nationale et bien sûr l'hebdomadaire le Yacht, très intéressé par toutes les questions maritimes :

61. Siegfried, André, Tableau politique de la France de l'Ouest sous la III République, Genève-Paris-Gex, Slatkine Reprints, 1980 (1éd., 1913), p. 222.

62. Le Finistère, le 10 décembre 1902.

63. La Dépêche de Brest, le 20 janvier 1903.

64. Le Courrier du Finistère, le 10 janvier 1903.

65. Archives de la Chambre de Commerce et d'Industrie de Brest, séance du 22 janvier 1903. 
" Nous avons tenu à rappeler très brièvement la situation, persuadés que les nombreux yachtmen qui ont pu dans leurs croisières apprécier les mérites de nos populations côtières auront à cœur de concourir au soulagement de leur grande détresse ${ }^{66}$."

Dès lors, c'est une concurrence de souscriptions, entre les différents journaux de la capitale et de province ${ }^{67}$. Le Gaulois offre un bon de cinq kilos de pain à 800 ménages; le Matin, l'lllustration, le Petit parisien ne sont pas en reste et proposent aux populations touchées des services équivalents. On note aussi dans les listes le nom de certains donateurs prestigieux. Le tsar Nicolas II verse 25000 francs, la reine Amélie du Portugal 3000 francs, les frères Rothschild 10000 francs; l'actrice Sarah Bernhardt verse la recette de la première de sa pièce Werther $^{68}$. Les communes de Marseille, de Toulouse ou de Rodez elles aussi participent au vaste mouvement de générosité. L'argent doit permettre de sauver les pêcheurs car " les plaintes échappées des hameaux bretons ont retenti au cœur des Parisiens que la charité ne trouva jamais insensible, et, à l'heure présente, il y a tout lieu de considérer la misère des sardiniers comme momentanément conjurée ${ }^{69}$ ".

Les conserveurs ne peuvent plus ignorer la détresse des marins qui vivent, à quelques encablures de leurs maisons et de leurs usines. À Douarnenez, les épouses Chancerelle et de Pénanros distribuent des tickets de pain et des pommes de terre achetées en grande quantité par leurs époux ou pères : "Ces messieurs continuent les achats de pommes de terre dont de grosses quantités auraient été déposées au Cercle catholique. Certaines craintes de discrimination se manifestent ${ }^{70}$. " Aux portes des usines Saupiquet ${ }^{71}$ et René Béziers au Guilvinec, sont servies des soupes chaudes; les Chancerelle distribuent des pommes de terre dans l'ancienne église qui n'est autre qu'un entrepôt leur ayant appartenu. Aux ouvrières de leurs friteries, les Nantais Philippe et Canaud offrent du pain tandis que le Concarnois Balestrié donne du chocolat et de la farine. Le maire et conserveur de Concarneau, Billette de Villeroche, lance l'idée d'une fête dont les bénéfices permettraient la création d'une œuvre destinée à aider les populations les plus touchées par la crise; le 10 septembre 1905, la première Fête des Filets Bleus se déroule dans le port concarnois, organisée par le poète Jos Parker et le peintre Gérard Legout ${ }^{72}$.

La dentelle aussi, dans une large mesure, pare à la crise sardinière et plus de 4000 ouvrières entre Camaret et le Pouldu s'adonnent à cette acti-

66. Le Yacht, 1903, p. 55.

67. Sellhac, Léon de, La Pêche de la sardine..., op. cit., p. 108.

68. LACHĖvRE, Yvon, La Sardine, toute une histoire, sl, Patrimoine maritime, 1994, p. 25.

69. SOUVESTRE, Pierre, dans Le Yacht, 1903, p. 68.

70. Arch. dép. du Finistère, 1 M 183, Quimper, le 3 octobre 1906, le commissaire spécial.

71. Carte postale d'époque légendée : «Une distribution de soupe à l'usine Saupiquet pendant la misère en Bretagne (à Kerity-Penmarc'h) ".

72. LE FLoc'H, Régis, Concarneau de 1852 à 1914, mémoire de maîtrise, UBO, Brest, 1993, p. 176 . 
vité en 1911 alors qu'elle était inconnue dix ans plus tôt ${ }^{73}$. Les veuves et épouses de conserveurs, mesdames Lacaze de Kerguvelen et Lemarié, se chargent de créer les premiers ateliers à Concarneau. Madame Delécluse, femme d'usinier elle aussi, réussit à fournir du travail à 200 femmes et jeunes filles de la région d'Audierne dès 1906. Mais dans ce cas encore les motifs philanthropiques proclamés à l'origine de la crise sont rapidement détournés et les dames patronnesses, dont bon nombre sont des proches des conserveurs, s'effacent au profit de négociants moins scrupuleux. L'industrie dentellière devait procurer un gagne-pain complémentaire à la population féminine côtière durement éprouvée par la disparition des sardines, mais " la mode aidant, l'industrie dentellière prit en même temps qu'une extension considérable un caractère plus mercantile ${ }^{74}$ ".

Malgré toutes ces querelles de chapelle, en dépit de la cacophonie ambiante, la générosité des Français est une réalité et la charité publique verse des millions. Il faut se préparer pour recueillir ces dons et les partager ensuite aux personnes nécessiteuses.

\section{Les deux Comités de secours ${ }^{75}$}

L'aide réclamée est votée le 23 janvier 1903, très rapidement, et il convient d'organiser, là aussi, la répartition de la somme, 500000 francs. La création du Comité officiel remonte au 9 janvier 1903, date à laquelle sont convoquées à la préfecture de Quimper les personnalités qualifiées pour venir en aide aux marins pêcheurs, mais aussi aux ouvriers et ouvrières d'usines que le " manque absolu de pêche avait plongés dans la plus profonde misère ${ }^{76}$ ". Sont jugés aptes à régler cette crise les députés et sénateurs du département, le préfet et les sous-préfets, les maires des chefs-lieux d'arrondissement, les conseillers généraux des cantons concernés, les maires des communes littorales entre Camaret et Clohars, les directeurs des journaux du département chargés de récupérer les souscriptions. À la tête du bureau on trouve les deux présidents d'honneur, l'amiral Roustan, préfet maritime de Bretagne, et Collignon, préfet du Finistère. James Monjarret de Kerjegu, député républicain de Quimperlé et président du conseil général est nommé président du Comité; Le Bail, député radical, et de Lécluse-Trévoëdal, maire catholique d'Audierne sont nommés vice-présidents. Toutes les préoccupations politiques sont très soigneusement et officiellement écartées des raisons de fonctionner du Comité lequel décide, lors de sa première assemblée générale, que

73. Arch. dép. du Finistère, 4 S 360, Rapport du Préfet du Finistère, non daté (octobre 1913).

74. Arch. dép. du Finistère, 10 M 9, Lorient, le 20 septembre 1911, l'inspecteur départemental du Travail, Châtelard.

75. Arch. dép. du Finistère, fonds $27 \mathrm{~J}$, documents du Comité de secours aux marins pêcheurs et ouvriers d'usines. Et pour le Comité catholique voir LÉOST, Alain, L'Épiscopat de Mgr Dubillard, mémoire de maîtrise d'histoire, Brest, UBO, 1990, p. 118-136.

76. Arch. dép. du Finistère, 27 J 23, lettre circulaire du préfet Collignon, Quimper, le 19 janvier 1903. 
l'organisation fasse " abstraction de toute politique ${ }^{77}$ " et que tous les fonds disponibles soient employés pour venir en aide aux marins, femmes et filles d'ouvriers d'usines malades et pour subventionner les sociétés de secours. En fait, dans un premier temps, il s'agit surtout de répartir équitablement entre une trentaine de communes plus de 100000 francs de dons déjà obtenus par les quotidiens finistériens et dans un second temps de tout faire :

" [...] Dans le but de recueillir des souscriptions en faveur des pêcheurs de sardines et des ouvriers d'usines plongés dans la plus profonde misère [...]. Ça représente plus de cent mille bouches sans pain. Il [le Comité] a décidé de faire appel à toute la France pour leur venir en aide ${ }^{78}$."

Le comité, par la voix de son président et du préfet, appelle l'évêque de Quimper, Mgr Dubillard, à le rejoindre pour faciliter l'envoi des dons, mais l'ecclésiastique refuse. Il n'a pas été invité à la première réunion du 9 janvier 1903 et n'apprécie pas cet affront ${ }^{79}$. Il annonce alors l'organisation d'un second comité, une bonne manière selon lui " d'aller aux marins " :

"Assurément j'applaudis, de toute mon âme, aux efforts qui sont faits pour venir en aide à nos pauvres pêcheurs, et je loue toutes les initiatives généreuses; mais il me sera permis de faire observer qu'aucun ecclésiastique, et ils sont nombreux dans notre ville, n'ayant été admis dans le Comité Central, j'ai dû craindre qu'il en fut de même dans les comités locaux ${ }^{80}$. "

Le Comité officiel reçoit la majorité des subsides et les 500000 francs octroyés par le parlement viennent très vite s'ajouter aux sommes recueillies; les dons arrivent de toute la France et même de plus loin. Les ambassades de France à Washington et à Moscou, les conseils municipaux de Paris, de Lyon, de Marseille mais aussi d'Orthez, de Tlemcen ou de Saïgon, réagissent et adressent leurs dons au Comité ${ }^{81}$. Ce dernier se trouve à la tête d'une véritable petite fortune. Par contre, les usiniers sont peu présents dans les listes des personnes charitables comme le souligne Le Finistère :

"Une seule chose nous surprend : c'est l'absence presque totale des fabricants de conserves sur les listes de souscription. Ils ont pourtant plus de raisons que n'importe qui pour venir en aide aux marins pêcheurs de nos côtes. Ces derniers ne sont-ils pas les principaux instruments de leur fortune ${ }^{82}$ ?"

Il semble que l'article sarcastique ait porté ses fruits car, quatre jours après sa publication, le syndicat des fabricants du Finistère et du Morbihan vote un don de 8000 francs pour le comité de secours, mais il est vrai que les usiniers ont plutôt collaboré avec le comité de l'évêque. Celui-ci reçoit des sommes envoyées par des catholiques, accompagnées souvent de lettres de protestation contre le gouvernement ou d'admiration pour

77. Arch. dép. du Finistère, 27 J 1, Quimper, le 9 janvier 1903.

78. Arch. dép. du Finistère, 27 J 1, "Ça " est souligné dans le texte par Le Bail.

79. Arch. dép. du Finistère, 27 J 5.

80. La Semaine Religieuse du diocèse de Quimper et de Léon du 23 janvier 1903.

81. Arch. dép. du Finistère, $27 \mathrm{~J} 1$.

82. Le Finistère, le 21 janvier 1903. 
Mgr Dubillard qui s'est opposé en août 1902 à l'expulsion des sœurs, les filles du Saint-Esprit. L'argent reçu est remis dans les paroisses sardinières les plus touchées pour qu'il soit distribué. Et les prêtres sont "à la hauteur, malgré d'énormes dépenses pour les pains et fourneaux : la suppression de [nos] traitements, faite avec une férocité inouïe, a valu à notre population beaucoup de dons particuliers, sans cela [nous] n'aur[ions] pu faire face à tant de dépenses ${ }^{83}$ ".

À la fin d'avril 1903, après trois mois d'existence, le comité diocésain a recueilli 222790 francs $^{84}$. Cinquante journaux royalistes et conservateurs ont transmis à Quimper les dons, de grands titres nationaux comme $L a$ Croix, La Libre Parole, le Gaulois, " qui jeta des wagons entiers d'aliments au milieu de ces populations désolées ${ }^{85}$ ", mais aussi des hebdomadaires locaux comme Le Nouvelliste de Bordeaux, Le Nouvelliste du Morbihan Le Patriote des Pyrénées, le Journal de la Meurthe, L'Émancipateur de Cambrai, Le Soleil du Midi... Il faut sauver à tout prix cette population bretonne dont on loue la " simplicité des mœurs et l'intensité de la foi ${ }^{86}$ " et dont on admire l'esprit de résistance. Les pêcheurs sardiniers et leur famille sont bien l'enjeu d'une lutte politique nationale et l'ampleur des dons permet de juger de l'intensité de la solidarité de chaque camp :

" Nos malheureux pêcheurs à qui la mer ne donnait plus de poisson se trouvèrent radicalement impuissants à se nourrir eux et leur famille. C'était littéralement la famine s'abattant sur nos côtes méridionales et atteignant une population de près de cent mille âmes qui vit exclusivement de la pêche de la sardine et du travail que procure sa préparation dans nos grands établissements industriels ${ }^{87}$.

C'est l'ensemble du monde catholique qui se mobilise, avec le soutien actif des papes, Léon XIII, décédé le 20 juillet 1903, puis Pie X. Le comité diocésain parvient à distribuer en deux ans près de 300000 francs, "grâce aux généreux concours des catholiques ${ }^{88}$ ". Rapidement les aides sont partagées par les prêtres " et nos bonnes religieuses ", à tous les pêcheurs des paroisses littorales, aussi bien à ceux de l'île de Sein où l'on ne pêche pas la sardine qu'à ceux de Molène ou de Fouesnant qui pratiquent essentiellement la pêche des crustacés ${ }^{89}$. Ce n'est donc plus la crise sardinière qui est au centre des débats, mais la misère de toute une profession. En

83. Archives de l'Évêché de Quimper, Penmarc'h, 1903, le recteur Le Coz à l'évêque. Cité dans Le Finistère 1800-1914 - les hommes, CDDP Finistère.

84. LÉOst, Alain, L'Épiscopat de Mgr Dubillard, op. cit., p. 121.

85. Arch. dép. du Finistère, 1 V 38, Quimper, 1903, instructions pastorales sur la charité chrétienne, Monseigneur Dubillard.

86. Arch. dép. du Finistère, 1 V 38, Quimper, 1900, lettre circulaire de Monseigneur Dubillard, évêque du diocèse.

87. Arch. dép. du Finistère, 1 V 38, Quimper, 1903, instructions pastorales sur la charité chrétienne, Monseigneur Dubillard.

88. Arch. dép. du Finistère, 1 V 86, Henri Bazire, Président du Comité national de l'Association de la Jeunesse catholique française, dans le Nouvelliste de Bretagne, le 24 mai 1904.

89. La Semaine Religieuse du diocèse de Quimper et de Léon, 23 janvier 1903. 
revanche, on peut remarquer que les aides sont largement distribuées dans le Finistère Nord, ou dans les îles, où le vote des marins pêcheurs est beaucoup plus favorable aux candidats catholiques que dans les grandes villes sardinières de Douarnenez ou de Concarneau plus hostiles.

Pour les catholiques, il est impensable de donner au Comité officiel où siègent des radicaux, les amis d'Émile Combes, un organisme forcément coupable de toutes les malversations, de tous les complots. Pour Paul Claudel, alors ambassadeur en Chine, il est inconcevable de donner au Comité officiel :

« J'ai lu avec beaucoup de douleur dans les journaux la relation de la misère navrante où sont actuellement plongés les pêcheurs de votre diocèse. Je serais très heureux de leur envoyer mon aumône, mais je ne veux à aucun prix me servir des comités officiels en qui je n'ai aucune confiance ${ }^{90}$."

Il faut dire que le fonctionnement du comité officiel est loin d'être des plus réguliers. Les vieilles rancunes personnelles et politiques prennent très tôt le pas sur la recherche de la meilleure répartition possible. Les discussions lors des réunions sont pour le moins houleuses; chacun des membres cherche à récupérer le maximum de fonds pour sa ville, son canton ou sa circonscription et chaque comité local agit de même. Tous ces hommes d'appareil qui " portent à son comble cette politique de recommandations, de patronages individuels, qu'on appelle la politique d'arrondissement ${ }^{91}$ ". Les observateurs sont unanimes pour condamner les attitudes partisanes : "Il fallait bien que la politique déshonorât ce magnifique mouvement de la solidarité générale par ses avilissantes querelles ${ }^{92}$ !"

De plus, les élus du Morbihan ne comprennent pas pourquoi ils sont exclus de ce comité dans la mesure où leur département est également touché par la crise, dans une moindre mesure il est vrai. Ils s'étonnent de la maigreur des subsides reçus, à peine 70000 francs, alors que leur voisin s'octroie 427300 francs des fonds publics gouvernementaux. Le comité finistérien se réunit très peu et les assemblées s'espacent de plus en plus avec le temps ${ }^{93}$ mais l'argent ne cesse de parvenir et il faut le distribuer, souvent dans la précipitation. D'ailleurs, le préfet Collignon demande l'arrêt des dons en argent, et par le décret du 21 novembre 1903 oblige à des distributions journalières en nature " d'aliments chauds par l'intermédiaire des fourneaux économiques ${ }^{94}$ ». En 1903, 622630 francs sont ainsi alloués aux « nécessiteux ", mais les caisses sont loin d'être vides ${ }^{95}$.

90. Paul Claudel à Fou Tchéou, Chine, le 20 janvier 1903, cité dans LÉost, Alain, L'Épiscopat de Mgr Dubillard..., op. cit., p. 126.

91. SIEGFRIED, André, Tableau politique..., op. cit., p. 148.

92. ROPERS, Louis, La Crise sardinière..., op. cit., 1906, p. 83.

93. Arch. dép. du Finistère, $27 \mathrm{~J}$ 1, Procès-verbaux des assemblées générales du Comité. 6 en 1903, 3 en 1904, 2 en 1905, une en 1908

94. Arch. dép. du Finistère, 27 J 6.

95. Création d'une institution de prévoyance et d'assistance en faveur de la pêche côtière \& des industries connexes dans le département du Finistère. Quimper, imprimerie du Finistère, 1903, p. 3. 
Plus de 2 millions de francs or ont été alloués entre 1903 et 1907 et les caisses du comité sont toujours largement garnies. Les fonds sont déposés à la Banque de France où ils produisent $3 \%$ d'intérêt annuel. Mais le fonctionnement quotidien du comité est pour le moins embrouillé, sinon secret, et il ne présente même pas de justificatifs de dépenses. Le rapport de la Cour des comptes est éloquent à ce sujet :

"La Cour a dû demander par injonction les quittances des créanciers réels et pour les secours en nature les mémoires des fournisseurs, pièces qui avaient été régulièrement produites dans les départements du Morbihan et de Vendée mais elle n'a pu obtenir que des états de répartition faisant connaître par commune les noms des bénéficiaires. Aucun de ces états ne porte l'émargement des parties prenantes [...] Il est même trois communes, celles de Douarnenez, Plouhinec et Guilvinec, où vu le grand nombre des bénéficiaires, les états nominatifs ont été remplacés par la simple indication du nombre de familles secourues et de la quantité approximative en partie prenante. Or la part de ces communes a été de 116715 francs, somme qui représente plus du quart de la dépense totale ${ }^{96}$."

Et pourtant, la distribution continue jusqu'en mars 1908, date à laquelle les membres du comité estiment que la situation des pêcheurs est bien meilleure et qu'elle ne nécessite plus une aide financière directe ${ }^{97}$. Il reste alors plus de 600000 francs en caisse que l'on destine aux sociétés de secours, aux caisses naissantes du Crédit Maritime ${ }^{98}$. Mais les réunions du comité sont tellement décousues qu'il devient impossible de prendre des décisions concrètes et fermes; de plus, la mort de Kerjégu en décembre 1908 fragilise un peu plus encore l'édifice. De nombreuses voix, externes et internes, s'élèvent pour condamner le Comité et surtout son mode de fonctionnement et de distribution. Pour beaucoup, il n'est qu'un appareil financier au seul service d'ambitions politiques. Le député Le Bail est particulièrement visé et attaqué pour son rôle prédominant au sein du comité. Le gouvernement lui-même est agacé par le fonctionnement si peu scrupuleux du comité et décide d'intervenir directement après le vote par le parlement d'un nouveau crédit de 500000 francs pour aider les pêcheurs et ouvriers touchés par la crise sardinière ${ }^{99}$ : le Finistère reçoit 350000 francs, le Morbihan 100000 et la Vendée, 50000, distribués par les agents de l'Inscription maritime.

De son côté le comité catholique a cessé d'exister. Le vote des lois d'expulsion des congrégations, de la séparation et des inventaires a sans doute assommé les meilleures volontés du diocèse, d'autant plus que le vote des

96. Arch. dép. du Finistère, 4 S 355, Paris le 30 août 1906, le ministre de l'Intérieur au préfet du Finistère.

97. Arch. dép. du Finistère, 27 J 1, procès-verbal de l'assemblée générale du 3 avril 1907. À cette date les recettes s'élèvent à 2328850 francs et les dépenses, dont les fonds placés, à 2326662 francs.

98. Arch. dép. du Finistère, 27 J 1, Procès-verbaux des assemblées générales du Comité.

99. Vote du 30 décembre 1909, crédit de secours provenant de la Caisse des Invalides. 
marins pêcheurs est loin de satisfaire un clergé sans doute déçu par l'attitude électorale de leurs ouailles. Pour conjurer le malheur apporté par le gouvernement Combes, il fallait tenir compte des " graves obligations du devoir électoral. Trop de Français, d'ailleurs bons chrétiens peut-être, les ignorent ou les oublient ${ }^{100}$ " et en particulier les marins pêcheurs concarnois ou douarnenistes.

Après six ans d'interruption, le comité se réunit de nouveau le 19 avril 1913. Que doit-on faire des 518369 francs dont il dispose à cette date? Plutôt que de reprendre les distributions qui ne satisfont personne, le comité décide d'aider la création d'une société coopérative de marins pêcheurs, "Les Pêcheries du Finistère " fondées en mai 1913. Au cours de la Grande Guerre, le comité est à nouveau muet avant de se réunir à nouveau le 14 août 1920 pour accorder 400000 francs en prêt à la caisse centrale du Crédit Maritime. Puis plus rien pendant sept ans jusqu'au 14 mars 1926, quand l'assemblée générale décide de liquider le Comité et de répartir les sommes disponibles. La dernière séance, " très agitée ", du 2 février 1929 se déroule " dans un véritable tumulte " après avoir distribué les derniers fonds ${ }^{101}$.

La crise sardinière commence en Bretagne méridionale à la fin de l'été 1902. Il s'agit à l'origine d'une simple crise d'approvisionnement, comme en connaissent fréquemment les pêcheurs de l'époque pour les poissons migrateurs, maquereaux, thons et sardines. Plus étonnant, cet accident majeur dure une douzaine d'années, avec des périodes de forte intensité et des passages beaucoup plus calmes, et sous des formes très variées, sociales, politiques économiques, culturelles... Il ne prend fin qu'avec le déclenchement de la Grande Guerre. Sans avoir jamais remis en cause la réalité de la misère initiale, les auteurs jusqu'à nos jours se sont contentés de présenter une misérable population de marins bretons sous ses aspects les plus caricaturaux. En fait, quand on reprend l'histoire de cette crise, on s'aperçoit qu'on peut l'analyser au prisme de la vision du monde selon les deux grands courants antagonistes qui s'affrontent au cours de cette période, catholiques concordataires et anticléricaux.

La disparition de la sardine divise la France non pas sous la forme convenue d'un épisode de la lutte des classes mais bien d'un combat entier entre deux branches de la population, toutes deux bien décidées à faire valoir leurs idées, d'ailleurs qu'elles soient en rapport avec la sardine ou non, en invoquant le nom de Dieu, qu'il soit maudit ou béni. Une partie de la Bretagne maritime est prompte à voir l'œuvre divine dans la disparition du précieux poisson, un avertissement après l'interdiction des congrégations religieuses, alors qu'une autre considère la politique anticléricale d'Émile

100. Arch. dép. du Finistère, 1 V 86, La Semaine Religieuse du diocèse de Quimper et de Léon du 5 janvier 1906.

101. Arch. dép. du Finistère, 27 J 1. Procès-verbaux des assemblées générales du Comité. 
Combes comme la marche à suivre pour obtenir satisfaction auprès des conserveurs jugés, à juste titre bien souvent, pour la plupart comme de puissants soutiens de l'Église catholique locale.

\section{RÉSUMÉ}

Au début de l'automne 1902, la sardine a disparu des côtes bretonnes et vendéennes; 15000 à 20000 ouvrières des conserveries, et le double de pêcheurs sont au chômage. La misère se fait tous les jours plus pesante. Pour tenter de la soulager, des fonds importants sont recueillis après une intense campagne de presse et un formidable élan national. Pour les distribuer, un comité de secours est constitué en janvier 1903, mais pour beaucoup de donateurs il présente le défaut d'être dirigé par des personnalités proches du gouvernement jugé trop républicain. Un second comité catholique est donc créé pour répondre aux attentes des donateurs hostiles aux radicaux et au gouvernement d'Émile Combes. Le grand débat d'idées à propos de la séparation de l'État et de l'Église qui secoue la France au cours des premières années du $\mathrm{Xx}^{\mathrm{e}}$ siècle trouve assez étonnamment un prolongement inattendu dans la grande crise sardinière qui frappe la Bretagne entre 1902 et 1907.

\section{ABSTRACT}

On early fall 1902, sardines disappeared from the coasts of Britanny and Vendée : 15 to 20000 women-workers from the sardine-curing stations, and about 30000 fishermen were out of work. Every day, misery grew heavier. To attempt to relieve it, important funds were gathered after an intense press campaign and a formidable national outburst of solidarity. To dispense the funds, a relief committee was created on January 1903, but its shortcoming, for many donors, was the fact that it was run by personalities who were too close to the Republican government. A second committee, more catholic, was consequently created to answer the expectations of anti-radical donors who were hostile to Emile Combes'government. The great debate that shook France during the early years of the $20^{\text {th }}$ Century found surprisingly its prolongation in the sardines crisis that stroke Britanny between 1902 and 1907. 